\title{
Backstepping-based estimation of thermoacoustic oscillations in a Rijke tube with experimental validation
}

\author{
Gustavo A. de Andrade, Rafael Vazquez, and Daniel J. Pagano
}

\begin{abstract}
We investigate the state observer design problem for thermoacoustic instabilities in a Rijke tube using an infinite dimensional perspective. The observer, whose design is based on the backstepping methodology with Volterra and full integral terms, consists of a copy of the linearized plant model plus output injection terms and relies only on a single boundary acoustic pressure sensor. The exponential convergence, in the $L_{2}$ sense, of the observed error dynamics is proved, and the analytic expression of the observer gains are derived explicitly. The results are tested in an experimental Rijke tube prototype in order to illustrate the effectiveness of the method.
\end{abstract}

Index Terms-Distributed parameter systems, Cascade systems, Observer design, thermoacoustic instability, Rijke tube.

\section{INTRODUCTION}

In this paper, we are concerned with the state estimation problem of thermoacoustic instabilities in a Rijke tube. This system consists of a vertical tube open at both ends (see Figure 1) with an embedded heat source (tipically a resistive heater). The air that traverses the heating zone expands, causing a sudden local pressure increase. The pressure acoustically propagates along the tube and returns, ultimately influencing itself at the heating area. A speaker placed under the tube is used as an actuator to suppress the oscillations, while a microphone at the top of the tube is used as sensor to provide the acoustic pressure measurements.

Thermoacoustic instability phenomena are often encountered in steam and gas turbines, industrial burners, and jet and ramjet engines. In such applications, the thermoacoustic instabilities are undesirable due to the vibrations resulting in mechanical failures, high levels of acoustic noise, high burn rates and component melting [1]. In this context, some studies have been focused on the development of automatic control strategies to stabilize these systems [2]. However, the control of such systems is complex due to the combustion reaction dynamics and its coupling with the acoustic pressure, which leads to a highly nonlinear behavior [3]. The Rijke tube on the other hand, is an academic experiment used for research and study of this phenomenon without needing a combustion process (see Figure 1) and still represents the thermoacoustic instabilities of industrial applications in a qualitative manner [4].

Gustavo A. de Andrade and Daniel J. Pagano are with the Departmento de Automação e Sistemas, Universidade Federal de Santa Catarina, 88040-370 Florianópolis, Brazil (e-mail: gustavo.artur@ufsc.br and daniel.pagano@ufsc.br, respectively).

Rafael Vazquez is with the Departmento de Ingeniería Aeroespacial, Universidad de Sevilla, 41092 Sevilla, Spain (e-mail: rvazquez1@us.es).

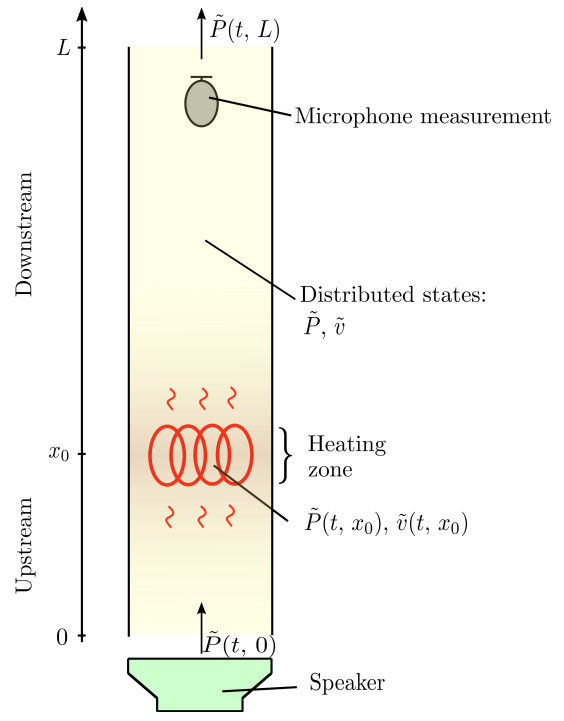

Fig. 1. Rijke tube diagram. Only the heater is necessary to induce the thermoacoustic instability. The microphone and speaker are used to measurement and actuation, respectivelly.

The thermoacoustic phenomenon in the Rijke tube can be modeled by linear first-order hyperbolic partial differential equations (PDEs) coupled with an ordinary differential equation (ODE) [3], [4]. Interestingly, this PDE system has a nonstrict-feedback connection with the ODE subsystem [5], which makes the application of standard control methodologies not possible (see [6] for more details). In this context, we have developed an approach to stabilize such system in [7], by employing the backstepping control design. However, the control law introduced in that work requires full-state measurements, which is not a realistic scenario. Thus, the use of a state observer together with the control law is the best option in order to apply that control design in a real-life experiment. This technical note is motivated by this challenge.

Many observer designs have been developed in literature to deal with the estimation of thermoacoustic instabilities. Approaches taking into account the distributed features of the system can be seen in [8], [9], [10], [11] and the references therein. However, these authors consider several simplifications in the mathematical modeling in order to obtain an expression suitable for standard control methodologies. In particular, it is only considered the downstream part of the tube and the measurements are assumed in a collocated fashion, which is not the general case. In this work, we take into 
account a more realistic model, including the heat release dynamics and both, the downstream and upstream parts of the tube, which results in a boundary PDE-ODE estimation problem.

Observer design for PDE-ODE cascade systems has been studied for many types of coupling, such as an ODE and diffusion PDEs [12], [13], [14], ODE and hyperbolic PDEs [15], [16], [17] and ODE and wave PDE [18]. The backstepping methodology plays the central role in this development [19], particularly for linear hyperbolic PDE-ODE systems [20], [21], [22], where the convergence properties of the system to the desired equilibrium point can be proved in exponential or finite time.

The main idea in the backstepping design is to use an invertible integral transformation (usually a Volterra transformation) to map the observer error dynamics into a target system with the desirable stability properties. This requires that the control engineer has reasonable knowledge about stability properties of PDEs. The advantage of this method is the obtaining of explicit state observer gains with simple structure. In addition, it does not employ model reductions, except at the implementation stage, where the system equations are solved numerically. In this context, we avoid spurious dynamics and erroneous predictions that can be induced by the model reduction methods. Nevertheless, due to the limitations of the standard Volterra transformation, most of the results in literature consider only strict-feedback connection with the ODE subsystem. Recently, some applications have appeared for very specific classes of systems with non-strict-feedback components [6], [23], [24], [25].

In this paper, a novel Luenberger-type state observer for estimation of thermoacoustic instabilities in the Rijke tube is proposed. The design is based on the backstepping methodology and relies only on one boundary acoustic pressure measurement. To tackle the non-strict-feedback form of the system, we formulate a well-posed backstepping transformation composed of Volterra and full integral terms that guarantee the exponential convergence of the observer to the actual values in the $L_{2}$ sense. The expression of the kernels of this transformation can be found explicitly, allowing us in turn to derive explicit observer gains. In particular, we show that the farther the heat element is from the pressure sensor, the more complex is the observer gains expression. Part of the results in this paper are contained in a preliminary form in [26] with much less details. Besides, we present here experimental results on a prototype of the Rijke tube.

The approach proposed in this paper can be particularly meaningful for the oil industry, such as drilling systems, where the distributed nature of the problem cannot be neglected and the mathematical models have similar structure to the one studied in this work [27]. Other applications include cascade delay-ODE systems, such as the motion of a piston in a viscous gas [28], solar thermal power plants [29], and photobioreactors [30].

The paper is organized as follows. In Section II we detail the system equations and formulate the state estimation problem. In Section III we design the observer gains using the backstepping method. Section IV presents results with experimental data from a Rijke tube prototype. Conclusions and future works are presented in Section V.

\section{PROBLEM DEFINITION}

The thermoacoustic phenomenon in Rijke tubes can be modeled by the following linearized equations (see [4] for a detailed derivation of the equations):

$$
\begin{aligned}
& \partial_{t} v(t, x)+\frac{1}{\bar{\rho}} \partial_{x} P(t, x)=0, \\
& \partial_{t} P(t, x)+\gamma \bar{P} \partial_{x} v(t, x)=\frac{\bar{\gamma}}{A} \delta\left(x-x_{0}\right) Q(t), \\
& \tau_{h r} \dot{Q}(t)+Q(t)=f^{\prime}(\bar{v})\left(T_{w}-\bar{T}_{g a s}\right) v\left(t, x_{0}\right),
\end{aligned}
$$

where $t \in[0,+\infty)$ is the time [s], $x \in[0, L]$ is the space [m], and $\delta$ is the Dirac delta distribution $[1 / \mathrm{m}]$. The distributed state $v$ stands for the velocity fluctuations $[\mathrm{m} / \mathrm{s}]$, and $P$ for the pressure fluctuations $[\mathrm{Pa}]$. The steady-state velocity $[\mathrm{m} / \mathrm{s}]$, density $\left[\mathrm{Kg} / \mathrm{m}^{3}\right]$ and pressure $[\mathrm{Pa}]$ are given by $\bar{v}, \bar{\rho}$ and $\bar{P}$, respectively. The tube cross-section area $\left[\mathrm{m}^{2}\right]$ is given by $A$, the heat capacity ratio is given by $\gamma=\frac{C_{P}}{C_{v}}$, where $C_{p}$ and $C_{v}$ are the specific heat capacity $[\mathrm{J} /(\mathrm{kg} \mathrm{K})]$ at constant pressure and volume conditions respectively, $x_{0}$ is the location of the heat release source $[\mathrm{m}], Q$ is the heat power release [W], $\tau_{h r}>0$ is the heat release time constant [s], $f(\bar{v})=l_{w}(\kappa+$ $\left.\kappa_{v} \sqrt{|\bar{v}|}\right)>0$ is the heat power transfer $[\mathrm{W} / \mathrm{K}], l_{w}$ is the wire length $[\mathrm{m}], \kappa$ is the fluid thermal conductivity $[\mathrm{W} /(\mathrm{m} \mathrm{K})], \kappa_{v}$ is an empirical constant $\left[\mathrm{W} \mathrm{s}^{1 / 2} /\left(\mathrm{m}^{1 / 2} \mathrm{~K}\right)\right]$, and $\bar{\gamma}=\gamma-1>0$. Finally, $T_{w}$ and $\bar{T}_{g a s}$, with $T_{w}-T_{g a s}>0$, stand for the wire and gas temperature $[\mathrm{K}]$, respectively.

System (1)-(3) is subjected to the boundary conditions

$$
P(t, 0)=U(t), \quad P(t, L)=Z_{L} v(t, L),
$$

where $Z_{L} \neq 0$ is the reflection loss $[\mathrm{Pa} \mathrm{s} / \mathrm{m}]$ and $U$ is the control variable $[\mathrm{Pa}]$.

Remark 1: Note that boundary conditions (4) imply that there are no acoustic pressure nodes in the tube's ends. This is in accordance with the prototype used to test the observer design proposed in this work. However, this cannot be generalized to all acoustic systems and each case must be analyzed carefully.

The initial condition of (1)-(4) is given by

$$
v(0, x)=v_{0}(x), \quad P(0, x)=P_{0}(x), \quad Q(0)=Q_{0},
$$

with $Q_{0} \in \mathbb{R}$ and $v_{0}, P_{0} \in \mathcal{L}_{2}([0, L])$.

The objective of this paper is to design an observer that provides accurate online estimates of both the finite-dimensional state $Q$ and the distributed state variables $P$ and $v$. The observer must only make use of the system input $U$ (although closed-loop control is not investigated in this paper) and output

$$
y(t)=P(t, L) .
$$

As a first step in our development, we reformulate the plant (1)-(4) in the form of transport PDEs. Using the Riemann coordinates

$$
\begin{aligned}
P(t, x) & =\frac{1}{2}\left(R_{1}(t, x)+R_{2}(t, x)\right), \\
v(t, x) & =\frac{1}{2 \sqrt{\gamma \bar{P} \bar{\rho}}}\left(R_{1}(t, x)-R_{2}(t, x)\right),
\end{aligned}
$$


system (1)-(4) can be rewritten as a $2 \times 2$ transport PDEs convecting in opposite directions with a point source term:

$$
\begin{aligned}
& \partial_{t} R_{1}(t, x)+c \partial_{x} R_{1}(t, x)=\frac{\bar{\gamma}}{A} \delta\left(x-x_{0}\right) Q(t), \\
& \partial_{t} R_{2}(t, x)-c \partial_{x} R_{2}(t, x)=\frac{\bar{\gamma}}{A} \delta\left(x-x_{0}\right) Q(t), \\
& \tau_{h r} \dot{Q}(t)=-Q(t)+q\left(R_{1}\left(t, x_{0}^{-}\right)-R_{2}\left(t, x_{0}^{+}\right)\right),
\end{aligned}
$$

where $c=\sqrt{\gamma \frac{\bar{P}}{\bar{\rho}}}$ is the speed of sound and $q=$ $\frac{f^{\prime}(\bar{v})\left(T_{w}-\bar{T}_{g a s}\right)}{2 \sqrt{\gamma \bar{P} \bar{\rho}}}$. In addition, the boundary conditions (4) are rewritten to

$$
R_{1}(t, 0)=-R_{2}(t, 0)+2 U(t), \quad R_{2}(t, L)=k R_{1}(t, L),
$$

with $k=\frac{Z_{L}-\bar{\rho} c}{Z_{L}+\bar{\rho} c}$.

From (7)-(8), we get that the following relations are satisfied:

$$
\begin{aligned}
& R_{1}\left(t, x_{0}^{+}\right)=R_{1}\left(t, x_{0}^{-}\right)+c_{1} Q(t) \\
& R_{2}\left(t, x_{0}^{-}\right)=R_{2}\left(t, x_{0}^{+}\right)+c_{1} Q(t),
\end{aligned}
$$

with $c_{1}=\frac{\bar{\gamma}}{A c}$

The jump relations (10)-(11) allows us to remove the point source term induced by the Dirac delta distribution by adding additional states and boundary conditions. To see that, define

$$
z= \begin{cases}\frac{x}{x_{0}}, & \text { if } x \in\left[0, x_{0}\right], \\ \frac{L-x}{L-x_{0}}, & \text { if } x \in\left[x_{0}, L\right]\end{cases}
$$

and consider

$$
\begin{array}{ll}
\alpha_{1}(t, x) \triangleq R_{1}(t, x), & \text { if } x \in\left[0, x_{0}\right], \\
\alpha_{2}(t, x) \triangleq R_{2}(t, x), & \text { if } x \in\left[x_{0}, L\right], \\
\beta_{1}(t, x) \triangleq R_{2}(t, x), & \text { if } x \in\left[0, x_{0}\right], \\
\beta_{2}(t, x) \triangleq R_{1}(t, x), & \text { if } x \in\left[x_{0}, L\right] .
\end{array}
$$

Then, the solution over each of the intervals $\left[0, x_{0}\right]$ and $\left[x_{0}, L\right]$ of (1)-(4), in characteristic coordinates, is equivalent to the following $4 \times 4 \mathrm{PDE}$ system, evolving in $z \in[0,1]$ :

$$
\begin{aligned}
& \partial_{t} \alpha_{1}(t, z)+\lambda_{1} \partial_{z} \alpha_{1}(t, z)=0, \\
& \partial_{t} \alpha_{2}(t, z)+\lambda_{2} \partial_{z} \alpha_{2}(t, z)=0, \\
& \partial_{t} \beta_{1}(t, z)-\lambda_{1} \partial_{z} \beta_{1}(t, z)=0, \\
& \partial_{t} \beta_{2}(t, z)-\lambda_{2} \partial_{z} \beta_{2}(t, z)=0, \\
& \tau_{h r} \dot{Q}(t)+Q(t)=q\left(\alpha_{1}(t, 1)-\alpha_{2}(t, 1)\right),
\end{aligned}
$$

with boundary conditions

$$
\begin{array}{ll}
\alpha_{1}(t, 0)=-\beta_{1}(t, 0), & \beta_{1}(t, 1)=\alpha_{2}(t, 1)+c_{1} Q(t), \\
\alpha_{2}(t, 0)=k \beta_{2}(t, 0), & \beta_{2}(t, 1)=\alpha_{1}(t, 1)+c_{1} Q(t),
\end{array}
$$

with , $\lambda_{1}=\frac{c}{x_{0}}, \lambda_{2}=\frac{c}{L-x_{0}}$. Note that $\lambda_{1}, \lambda_{2}, c_{1}, q>0$.

Remark 2: If $x_{0} \leq \frac{1}{2} L$, i.e., if the heat release is located in the lower half of the tube, then $\lambda_{1}<\lambda_{2}$, otherwise $\lambda_{1} \geq \lambda_{2}$.

Besides, using (5) and (12) we get that

$$
y(t)=\frac{Z_{L}}{Z_{L}+\bar{\rho} c} \beta_{2}(t, 0) .
$$

In this new framework, the thermoacoustic phenomenon is represented as the cascade of four transport PDEs and one ODE being driven by (13)-(14). In the next section we derive an observer based on these equations.

\section{OBSERVER DESIGN AND ERROR DYNAMICS ANALYSIS}

\section{A. Observer design}

Let $\hat{\alpha}_{i}$ and $\hat{\beta}_{i}$, for $i \in\{1,2\}$, and $\hat{Q}$ be the estimated states. We design the observer as a copy of the plant (13)-(19) plus output injection terms, i.e.,

$$
\begin{aligned}
& \partial_{t} \hat{\alpha}_{1}(t, z)+\lambda_{1} \partial_{z} \hat{\alpha}_{1}(t, z)=-p_{1}(z) \tilde{Y}(t), \\
& \partial_{t} \hat{\alpha}_{2}(t, z)+\lambda_{2} \partial_{z} \hat{\alpha}_{2}(t, z)=0, \\
& \partial_{t} \hat{\beta}_{1}(t, z)-\lambda_{1} \partial_{z} \hat{\beta}_{1}(t, z)=-p_{2}(z) \tilde{Y}(t), \\
& \partial_{t} \hat{\beta}_{2}(t, z)-\lambda_{2} \partial_{z} \hat{\beta}_{2}(t, z)=-p_{3}(z) \tilde{Y}(t), \\
& \tau_{h r} \dot{\hat{Q}}(t)+\hat{Q}(t)=q\left(\hat{\alpha}_{1}(t, 1)-\hat{\beta}_{2}(t, 1)\right)-p_{Q} \tilde{Y}(t),
\end{aligned}
$$

where $\tilde{Y}(t)=\beta_{2}(t, 0)-\hat{\beta}_{2}(t, 0)$, and $p_{1}, p_{2}, p_{3}$, and $p_{Q}$ are gains to be found. The boundary conditions of (21)-(25) are given by

$$
\begin{array}{ll}
\hat{\alpha}_{1}(t, 0)=-\hat{\beta}_{1}(t, 0), & \hat{\beta}_{1}(t, 1)=\hat{\alpha}_{2}(t, 1)+c_{1} \hat{Q}(t), \\
\hat{\alpha}_{2}(t, 0)=k \beta_{2}(t, 0), & \hat{\beta}_{2}(t, 1)=\hat{\alpha}_{1}(t, 1)+c_{1} \hat{Q}(t) .
\end{array}
$$

Note that the real value of $\beta_{2}(t, 0)$ is directly applied into the boundary condition of $\hat{\alpha}_{2}(t, 0)$ in (27) since this information is always available through the output defined in (20).

\section{B. Target system}

Define the estimation error as $\tilde{\alpha}_{i}=\alpha_{i}-\hat{\alpha}_{i}, \tilde{\beta}_{i}=\beta_{i}-\hat{\beta}_{i}$, for $i \in\{1,2\}$, and $\tilde{Q}=Q-\hat{Q}$. Then, using (13)-(19) and (21)-(27), we obtain the following error dynamics:

$$
\begin{aligned}
& \partial_{t} \tilde{\alpha}_{1}(t, z)+\lambda_{1} \partial_{z} \tilde{\alpha}_{1}(t, z)=p_{1}(z) \tilde{Y}(t), \\
& \partial_{t} \tilde{\alpha}_{2}(t, z)+\lambda_{2} \partial_{z} \tilde{\alpha}_{2}(t, z)=0, \\
& \partial_{t} \tilde{\beta}_{1}(t, z)-\lambda_{1} \partial_{z} \tilde{\beta}_{1}(t, z)=p_{2}(z) \tilde{Y}(t), \\
& \partial_{t} \tilde{\beta}_{2}(t, z)-\lambda_{2} \partial_{z} \tilde{\beta}_{2}(t, z)=p_{3}(z) \tilde{Y}(t), \\
& \tau_{h r} \dot{\tilde{Q}}(t)+\tilde{Q}(t)=q\left(\tilde{\alpha}_{1}(t, 1)-\tilde{\beta}_{2}(t, 1)\right)+p_{Q} \tilde{Y}(t),
\end{aligned}
$$

with boundary conditions

$$
\begin{array}{ll}
\tilde{\alpha}_{1}(t, 0)=-\tilde{\beta}_{1}(t, 0), & \tilde{\beta}_{1}(t, 1)=\tilde{\alpha}_{2}(t, 1)+c_{1} \tilde{Q}(t), \\
\tilde{\alpha}_{2}(t, 0)=0, & \tilde{\beta}_{2}(t, 1)=\tilde{\alpha}_{1}(t, 1)+c_{1} \tilde{Q}(t) .
\end{array}
$$

The coupling between the boundaries (33)-(34) and the ODE (32) can potentially leads to an unstable behavior (see more details in [7]). Therefore, we design the observer output injection gains $p_{1}, p_{2}, p_{3}$, and $p_{Q}$, such that (28)-(34) is mapped to the following target system:

$$
\begin{aligned}
& \partial_{t} \check{\alpha}_{1}(t, z)+\lambda_{1} \partial_{z} \check{\alpha}_{1}(t, z)=0, \\
& \partial_{t} \check{\alpha}_{2}(t, z)+\lambda_{2} \partial_{z} \check{\alpha}_{2}(t, z)=0 \text {, } \\
& \partial_{t} \check{\beta}_{1}(t, z)-\lambda_{1} \partial_{z} \check{\beta}_{1}(t, z)=0 \text {, } \\
& \partial_{t} \check{\beta}_{2}(t, z)-\lambda_{2} \partial_{z} \check{\beta}_{2}(t, z)=0 \text {, } \\
& \tau_{h r} \dot{\mathscr{Q}}(t)+\left(1+c_{1} q\right) \check{Q}(t)=-q \check{\alpha}_{2}(t, 1) \text {, }
\end{aligned}
$$

with boundary conditions

$$
\begin{array}{lll}
\check{\alpha}_{1}(t, 0)=-\check{\beta}_{1}(t, 0), & \check{\beta}_{1}(t, 1)=\check{\alpha}_{2}(t, 1)+c_{1} \check{Q}(t), \\
\check{\alpha}_{2}(t, 0)=0, & \check{\beta}_{2}(t, 1)=\check{\alpha}_{1}(t, 1)+c_{1} \check{Q}(t),
\end{array}
$$


System (35)-(41) is a copy of the original error dynamics, from which the ODE (32) has been changed in order to achieve exponential stability, according to the following definition:

Definition 1: The hyperbolic system (35)-(41) is exponentially stable, if there exist $\nu>0$, and $C>0$ such that, for every initial condition $\left(\check{\alpha}_{1}^{0}, \check{\alpha}_{2}^{0}, \check{\beta}_{1}^{0}, \check{\beta}_{2}^{0}\right) \in\left(\mathcal{L}_{2}([0,1])\right)^{4}$, the solution of the problem (35)-(41) satisfies

$$
\begin{aligned}
\left\|\left(\check{\alpha}_{1}^{0}, \check{\alpha}_{2}^{0}, \check{\beta}_{1}^{0}, \check{\beta}_{2}^{0}\right)\right\|_{\left(\mathcal{L}_{2}([0,1])\right)^{4}+|Q| \leq} \\
\\
C \mathrm{e}^{-\nu t}\left(\left\|\left(\check{\alpha}_{1}^{0}, \check{\alpha}_{2}^{0}, \check{\beta}_{1}^{0}, \check{\beta}_{2}^{0}\right)\right\|_{\left(\mathcal{L}_{2}([0,1])\right)^{4}}+\left|Q_{0}\right|\right),
\end{aligned}
$$

for all $t \in[0, \infty)$.

Lemma 1: Consider system (35)-(39) with boundary conditions (40)-(41) and initial condition $\check{\alpha}_{1}^{0}, \check{\alpha}_{2}^{0}, \breve{\beta}_{1}^{0}, \check{\beta}_{2}^{0} \in$ $\mathcal{L}_{2}([0,1], \mathbb{R})$ and $\check{Q}^{0} \in \mathbb{R}$. In addition, consider that $\tau_{h r}, \lambda_{1}, \lambda_{2}, q, c_{1}>0$. Then, its zero equilibrium is exponentially stable in the $\mathcal{L}_{2}$ sense.

Proof: Consider the following candidate Lyapunov functional:

$$
\begin{aligned}
& V(t)=\frac{\tau_{h r}}{2} \sigma_{0} Q^{2}(t)+\frac{1}{2} \int_{0}^{1}\left[\sigma_{1} \mathrm{e}^{-z} \alpha_{1}^{2}(t, z)\right. \\
& \left.+\sigma_{2} \mathrm{e}^{-z} \alpha_{2}^{2}(t, z)+\sigma_{3} \mathrm{e}^{z} \beta_{1}^{2}(t, z)+\mathrm{e}^{z} \beta_{2}^{2}(t, z)\right] d z
\end{aligned}
$$

where $\sigma_{i}, i \in\{0, \ldots, 3\}$, are positive constants to be determined.

Define $\alpha_{i}(0) \triangleq \alpha_{i}(t, 0), \beta_{i}(0) \triangleq \beta_{i}(t, 0), \alpha_{i}(1) \triangleq$ $\alpha_{i}(t, 1), \beta_{i}(1) \triangleq \beta_{i}(t, 1)$, for $i \in\{1,2\}$. Then, differentiating (42) with respect to time, integrating by parts and substituting the boundary conditions (40)-(41) yields

$$
\begin{array}{r}
\dot{V}(t)=Q^{2}(t)\left[\frac{1}{2} \mathrm{e}^{1} c_{1}^{2}\left(\sigma_{3} \lambda_{1}+\lambda_{2}\right)-\sigma_{0}\left(1+c_{1} q\right)\right] \\
+\frac{1}{2} \lambda_{1}\left(\sigma_{1}-\sigma_{3}\right) \beta_{1}^{2}(0)+\frac{1}{2}\left(\lambda_{2} \mathrm{e}^{1}-\lambda_{1} \sigma_{1} \mathrm{e}^{-1}\right) \alpha_{1}^{2}(1) \\
+\frac{1}{2}\left(\lambda_{1} \sigma_{3} \mathrm{e}^{1}-\lambda_{2} \sigma_{2} \mathrm{e}^{-1}\right) \alpha_{2}^{2}(1)-\frac{1}{2} \lambda_{2} \beta_{2}^{2}(0) \\
+\left(\lambda_{1} \sigma_{3} \mathrm{e}^{1} c_{1}-\alpha_{0} q\right) \alpha_{2}(1) Q(t)+c_{1} \lambda_{2} \mathrm{e}^{1} \alpha_{1}(1) Q(t) \\
-\frac{1}{2} \int_{0}^{1}\left[\sigma_{1} \mathrm{e}^{-z} \alpha_{1}^{2}(t, z)+\sigma_{2} \mathrm{e}^{-z} \alpha_{2}^{2}(t, z)+\mathrm{e}^{z} \beta_{1}(t, z)\right. \\
\left.+\mathrm{e}^{z} \beta_{2}^{2}(t, z)\right] d z .
\end{array}
$$

Let $\sigma_{3}>\sigma_{1}$ and $\sigma_{2}=\frac{\sigma_{3} \lambda_{1} \mathrm{e}^{1}}{\lambda_{2} \mathrm{e}^{-1}}+\frac{\sigma_{4}}{\lambda_{2} \mathrm{e}^{-1}}$ for some positive constant $\sigma_{4}$. It follows that

$$
\begin{gathered}
\dot{V}(t) \leq Q^{2}(t)\left[\frac{1}{2} c_{1}^{2} \lambda_{1} \sigma_{3} \mathrm{e}^{1}-\sigma_{0}\left(1+c_{1} q\right)\right] \\
+\frac{1}{2} \alpha_{1}^{2}(1)\left[\lambda_{2} \mathrm{e}^{1}+\frac{\left(-\lambda_{1} c_{1} \mathrm{e}^{-1}+\lambda_{2} \mathrm{e}^{1}\right)^{2}}{\lambda_{2} \mathrm{e}^{1} c_{1}^{2}}-\lambda_{1} \sigma_{1} \mathrm{e}^{-1}\right] \\
+\frac{1}{2} \alpha_{2}^{2}(1)\left(\frac{\lambda_{2} c_{1}^{2} \mathrm{e}^{1}}{\lambda_{1} c_{1} \sigma_{3} \mathrm{e}^{1}-q \sigma_{0}}-\sigma_{4}\right)-\frac{1}{2} \int_{0}^{1}\left[\sigma_{1} \mathrm{e}^{-z} \alpha_{1}^{2}(t, z)\right. \\
\left.+\sigma_{2} \mathrm{e}^{-z} \alpha_{2}^{2}(t, z)+\mathrm{e}^{z} \beta_{1}(t, z)+\mathrm{e}^{z} \beta_{2}^{2}(t, z)\right] d z .
\end{gathered}
$$

Thus, choosing

$$
\begin{aligned}
\sigma_{0} & >\frac{1}{2} \frac{\lambda_{1} c_{1}^{2} \sigma_{3} \mathrm{e}^{1}}{c_{1}(1+q)}, \\
\sigma_{1} & >\frac{\lambda_{2} \mathrm{e}^{1}}{\lambda_{1} \mathrm{e}^{-1}}+\frac{\left(\lambda_{2} \mathrm{e}^{1}-\lambda_{1} c_{1} \mathrm{e}^{-1}\right)^{2}}{\lambda_{1} \lambda_{2} c_{1}^{2} \mathrm{e}^{1}}, \\
\sigma_{4} & >\frac{\lambda_{2} c_{1}^{2} \mathrm{e}^{1}}{\lambda_{1} c_{1} \sigma_{3} \mathrm{e}^{1}-q \sigma_{0}},
\end{aligned}
$$

we have the announced result.

\section{Backstepping transformation}

To map system (28)-(34) into (35)-(41), we consider the following backstepping transformation:

$$
\begin{aligned}
\tilde{\alpha}_{1}(t, z) & =\check{\alpha}_{1}(t, z)-\int_{0}^{1} P_{1}(z, \xi) \check{\beta}_{2}(t, \xi) d \xi, \\
\tilde{\beta}_{1}(t, z) & =\check{\beta}_{1}(t, z)-\int_{0}^{1} P_{2}(z, \xi) \check{\beta}_{2}(t, \xi) d \xi, \\
\tilde{\beta}_{2}(t, z) & =\check{\beta}_{2}(t, z)-\int_{0}^{z} P_{3}(z, \xi) \check{\beta}_{2}(t, \xi) d \xi, \\
\tilde{Q}(t) & =\check{Q}(t)-\int_{0}^{1} P_{Q}(\xi) \check{\beta}_{2}(t, \xi) d \xi,
\end{aligned}
$$

where $P_{i}, i \in\{1,2,3\}$, and $P_{q}$ are the kernels to be determined such that (44)-(47) maps system (28)-(34) into (35)-(41).

Differentiating (44)-(47) with respect to space and time, plugging the target system equations (35)-(41) and integrating by parts, we obtain that (28)-(34) is mapped into (35)-(41) if and only if (see [20] for more details) the kernels satisfy the following equations:

$$
\begin{aligned}
& \lambda_{2} \partial_{\xi} P_{1}(z, \xi)-\lambda_{1} \partial_{z} P_{1}(z, \xi)=0, \\
& \lambda_{2} \partial_{\xi} P_{2}(z, \xi)+\lambda_{1} \partial_{z} P_{2}(z, \xi)=0, \\
& \partial_{\xi} P_{3}(z, \xi)+\partial_{z} P_{3}(z, \xi)=0, \\
& \tau_{h r} \lambda_{2} P_{Q}^{\prime}(\xi)-P_{Q}(\xi)=-q P_{1}(1, \xi),
\end{aligned}
$$

with boundary conditions

$$
P_{1}(z, 1)=0, \quad P_{2}(z, 1)=0, \quad P_{Q}(1)=-\frac{q}{\tau_{h r} \lambda_{2}} .
$$

Evaluating (44) for $z=0$ and (45)-(46) for $z=1$, and using the boundary conditions (33)-(34), we have the following three additional conditions:

$$
\begin{aligned}
& P_{1}(0, \xi)=-P_{2}(0, \xi), \\
& P_{3}(1, \xi)=P_{1}(1, \xi)+c_{1} P_{Q}(\xi) .
\end{aligned}
$$

The observer gains are given by

$$
\begin{aligned}
p_{1}(z) & =\lambda_{2} P_{1}(z, 0), & p_{2}(z) & =\lambda_{2} P_{2}(z, 0), \\
p_{3}(z) & =\lambda_{2} P_{3}(z, 0), & p_{Q} & =\tau_{h r} \lambda_{2} P_{Q}(0) .
\end{aligned}
$$

Lemma 2: Let $\tau_{h r}, \lambda_{1}, \lambda_{2}, q, c_{1}>0$. Then, system (48)-(54) has a unique solution in $\mathcal{L}^{\infty}([0,1])$.

The proof of this lemma is established in the next section. In particular we find an explicit solution for the kernel equations which is piecewise differentiable. 


\section{Well-posedness and explicit solution of the kernel equa-} tions

In this section, we investigate the existence and uniqueness of the solution of the kernel equations (48)-(54). Since these equations have a simple structure, we study a closed solution to them by using the method of characteristics (see [31] for more details). Although this approach to solving firstorder hyperbolic PDEs is classical, the particular geometry of the problem and the coupling between kernels could lead to unbounded solutions on finite intervals.

Solution for $P_{1}$ kernel. For all $(z, \xi) \in[0,1] \times[0,1]$ consider the following characteristic lines:

$$
\chi_{1}(s ; z)=-\lambda_{1} s+z, \quad \zeta_{1}(s ; \xi)=\lambda_{2} s+\xi .
$$

Integrating (48) along (57), using the fact that $P_{1}$ is constant along these lines, and plugging the boundary conditions (52) and (53), we obtain the following solution:

$$
P_{1}(z, \xi)= \begin{cases}0, & \frac{\lambda_{1}}{\lambda_{2}}(1-\xi) \geq z, \\ -P_{2}\left(0, \xi+\frac{\lambda_{2}}{\lambda_{1}} z\right), & \text { otherwise. }\end{cases}
$$

Solution for $P_{2}$ kernel. In this case, we define the following characteristic lines

$$
\chi_{2}(s ; z)=-\lambda_{1} s+z, \quad \zeta_{2}(s ; \xi)=-\lambda_{2} s+\xi,
$$

for all $(z, \xi) \in[0,1] \times[0,1]$. Then, integrating (49) along (59), noting that $P_{2}$ is constant along these lines, and plugging the boundary conditions (52) and (53), we obtain

$P_{2}(z, \xi)= \begin{cases}0, & \frac{\lambda_{1}}{\lambda_{2}}(\xi-1) \geq z-1, \\ c_{1} P_{Q}\left(\xi-\frac{\lambda_{2}}{\lambda_{1}}(z-1)\right), & \text { otherwise. }\end{cases}$

Solution for $P_{3}$ kernel. For $(z, \xi) \in\{0 \leq \xi \leq z \leq 1\}$, we define the following characteristic lines corresponding to (50):

$$
\chi_{3}(s ; z)=-s+z, \quad \zeta_{3}(s ; \xi)=-s+\xi .
$$

Integrating (50) along (61), using the fact that $P_{3}$ is constant along these lines, and plugging boundary condition (54), we have that

$$
P_{3}(z, \xi)=P_{1}(1,1-z+\xi)+c_{1} P_{Q}(1-z+\xi) .
$$

Solution for $P_{Q}$ kernel. As can be seen in (51), the $P_{Q}$ kernel equation depends explicitly of $P_{1}$, which may have a discontinuity at $\lambda_{1} \xi+\lambda_{2} z=\lambda_{1}$, when $\lambda_{1} \geq \lambda_{2}$ (see Remark 2 for a physical interpretation of this inequality). When such case occurs, the kernel equation structure allows us to construct the solution in a stepwise fashion, as we show in the next lemma.

Lemma 3: Consider ODE (51) with boundary condition (52), such that $P_{1}$ and $P_{2}$ are defined by (58) and (60), respectively. Then:

(I) If $\lambda_{1} \leq \lambda_{2}$, the solution of (51) with boundary condition (52) is given by

$$
P_{Q}(\xi)=-\frac{q}{\tau_{h r} \lambda_{2}} \mathrm{e}^{\frac{1}{\tau_{h r} \lambda_{2}}(\xi-1)} .
$$

(II) If $\lambda_{1}>\lambda_{2}$, the solution of (51) with boundary condition (52) is given

$$
P_{Q}(\xi)= \begin{cases}P_{Q, 0}(\xi), & \text { if } \xi \in \Omega_{0} \\ \vdots & \\ P_{Q, n}(\xi), & \text { if } \xi \in \Omega_{n}\end{cases}
$$

with

$$
\Omega_{k}=\left\{\xi \in[0,1]: 1-\frac{2^{k+1} \lambda_{2}}{\lambda_{1}} \leq \xi \leq 1-\frac{2^{k} \lambda_{2}}{\lambda_{1}}\right\},
$$

where $k=1, \ldots, n$ and $n$ is the largest integer such that $\frac{\lambda_{2}}{\lambda_{1}}<\frac{1}{2^{n}}$. In addition, $P_{Q, 0}(\xi)=$ $-\frac{q}{\tau_{h r} \lambda_{2}} \mathrm{e}^{\frac{1}{\tau_{h r} \lambda_{2}}(\xi-1)}$ and

$$
\begin{gathered}
P_{Q, k}(\xi)=\left[\mathrm{e}^{\frac{2^{k}}{\tau_{h r} \lambda_{1}}} P_{Q, k-1}\left(1-\frac{2^{k} \lambda_{2}}{\lambda_{1}}\right)+\frac{q c_{1}}{\tau_{h r} \lambda_{2}} \times\right. \\
\left.\int_{\xi}^{1-\frac{2^{k} \lambda_{2}}{\lambda_{1}}} \mathrm{e}^{-\frac{\theta-1}{\tau_{h r} \lambda_{2}}} P_{Q, k-1}\left(\theta+2 \frac{\lambda_{2}}{\lambda_{1}}\right)\right] \mathrm{e}^{\frac{1}{\tau_{h r} \lambda_{2}}}(\xi-1)
\end{gathered}
$$

for $k=1, \ldots, n$.

Proof: We will show this result for each case separately:

Case I $\left(\lambda_{1} \leq \lambda_{2}\right)$ : In this case, $P_{2}\left(0, \xi+\frac{\lambda_{2}}{\lambda_{1}} z\right)=0$ for all $(z, \xi) \in[0,1] \times[0,1]$ and therefore $P_{1}(z, \xi)=0$ for all $(z, \xi) \in[0,1] \times[0,1]$. Then, it follows that the $P_{Q}$ kernel equation (51) is simplified to

$$
\tau_{h r} \lambda_{2} P_{Q}^{\prime}(\xi)=P_{Q}(\xi) .
$$

Therefore, integrating the above equation and plugging (54) yields the following solution:

$$
P_{Q}(\xi)=-\frac{q}{\tau_{h r} \lambda_{2}} \mathrm{e}^{\frac{1}{\tau_{h r} \lambda_{2}}(\xi-1)} .
$$

Case II $\left(\lambda_{1}>\lambda_{2}\right)$ : We will show this result by induction. First, note that $P_{1}(1, \xi)=0$ for all $\xi \in\left[1-2 \frac{\lambda_{2}}{\lambda_{1}}, 1\right]$ and therefore, the solution of $P_{Q}$ for $\xi \in\left[1-2 \frac{\lambda_{2}}{\lambda_{1}}, 1\right]$ satisfies the nonautonomous ODE (67) with boundary condition (54). As shown above, this equation has a unique solution, given by (68), and for all $\xi \in \Omega_{0}$ with $\lambda_{1}>\lambda_{2}$ the result holds for $k=0$.

Consider that $k=m$, with $m \in \mathbb{N}$, and that (64)-(66) hold. Accordingly, we assume that

$$
\begin{aligned}
& P_{Q}(\xi)=\left[\mathrm{e}^{\frac{2^{m}}{\tau_{h r} \lambda_{1}}} P_{Q, m-1}\left(1-\frac{2^{m} \lambda_{2}}{\lambda_{1}}\right)+\frac{q c_{1}}{\tau_{h r} \lambda_{2}} \times\right. \\
& \left.\int_{\xi}^{1-\frac{2^{m} \lambda_{2}}{\lambda_{1}}} \mathrm{e}^{-\frac{\theta-1}{\tau_{h r} \lambda_{2}}} P_{Q, m-1}\left(\theta+2 \frac{\lambda_{2}}{\lambda_{1}}\right) d \theta\right] \mathrm{e}^{\frac{1}{\tau_{h r} \lambda_{2}}(\xi-1)}
\end{aligned}
$$

is the solution of the following boundary value problem:

$$
\left\{\begin{array}{l}
\tau_{h r} \lambda_{2} P_{Q}^{\prime}(\xi)=P_{Q}(\xi)-q P_{Q, m-1}\left(\xi+2 \frac{\lambda_{2}}{\lambda_{1}}\right) \\
P_{Q}\left(1-2^{m} \frac{\lambda_{2}}{\lambda_{1}}\right)=P_{Q, m-1}\left(1-2^{m} \frac{\lambda_{2}}{\lambda_{1}}\right)
\end{array}\right.
$$


over the interval $\Omega_{m}$.

Now, for $k=m+1$, we have

$$
\left\{\begin{array}{l}
\tau_{h r} \lambda_{2} P_{Q}^{\prime}(\xi)=P_{Q}(\xi)-q P_{Q, m}\left(\xi+2 \frac{\lambda_{2}}{\lambda_{1}}\right) \\
P_{Q}\left(1-2^{m+1} \frac{\lambda_{2}}{\lambda_{1}}\right)=P_{Q, m}\left(1-2^{m+1} \frac{\lambda_{2}}{\lambda_{1}}\right)
\end{array}\right.
$$

Integrating this expression from $\xi$ to $1-2^{m+1} \frac{\lambda_{2}}{\lambda_{1}}$, after some computations, we obtain

$$
\begin{aligned}
& P_{Q}(\xi)=\left[\mathrm{e}^{\frac{2^{m+1}}{\tau_{h r} \lambda_{1}}} P_{Q, m}\left(1-\frac{2^{m+1} \lambda_{2}}{\lambda_{1}}\right)+\frac{q c_{1}}{\tau_{h r} \lambda_{2}} \times\right. \\
& \left.\int_{\xi}^{1-\frac{2^{m+1} \lambda_{2}}{\lambda_{1}}} \mathrm{e}^{-\frac{\theta-1}{\tau_{h r} \lambda_{2}}} P_{Q, m}\left(\theta+2 \frac{\lambda_{2}}{\lambda_{1}}\right)\right] \mathrm{e}^{\frac{1}{\tau_{h r} \lambda_{2}}(\xi-1)},
\end{aligned}
$$

and hence (66) is also valid for $k=m+1$. Hence, by mathematical induction the result holds. This concludes the proof.

Remark 3: Note that the number of pieces of (66) is finite and depends on the values of $\lambda_{1}$ and $\lambda_{2}$ (see Remark 2).

\section{E. Inverse transformation}

To ensure that the target system (35)-(41) and the observer error system (28)-(34) have equivalent stability properties, transformation (44)-(47) has to be invertible. Since $P_{3}$ is piecewise-differentiable and bounded, and (46) is a scalar Volterra integral equation of the second type, then there exists a unique inverse kernel $L_{3}$ such that [32]

$$
\check{\beta}_{2}(t, z)=\tilde{\beta}_{2}(t, z)+\int_{0}^{z} L_{3}(z, \xi) \tilde{\beta}_{2}(t, \xi) d \xi .
$$

Plugging (71) into (44)-(45) and (47) yields

$$
\begin{aligned}
\check{\alpha}_{1}(t, z) & =\tilde{\alpha}_{1}(t, z)+\int_{0}^{1} L_{1}(z, \xi) \tilde{\alpha}_{1}(t, \xi) d \xi \\
\check{\beta}_{1}(t, z) & =\tilde{\beta}_{1}(t, z)+\int_{0}^{1} L_{2}(z, \xi) \tilde{\beta}_{1}(t, \xi) d \xi \\
\tilde{Q}(t) & =\check{Q}+\int_{0}^{1} L_{Q}(\xi) \check{Q}(\xi) d \xi
\end{aligned}
$$

where

$$
\begin{gathered}
L_{1}(z, \xi)=P_{1}(z, \xi)+\int_{\xi}^{1} P_{1}(z, \xi) L_{3}(\xi, s) d s, \\
L_{2}(z, \xi)=P_{2}(z, \xi)+\int_{\xi}^{1} P_{2}(z, \xi) L_{3}(\xi, s) d s, \\
L_{Q}(\xi)=P_{Q}(\xi)+\int_{\xi}^{1} P_{Q}(1) L_{3}(\xi, s) d s .
\end{gathered}
$$

Note that (75)-(77) are well-posed Volterra integral equations of the second type, since $L_{3}$ is well-posed. Therefore, one finds that there is a unique solution to (72)-(74) and thus the invertibility of the transformation is proved.

\section{F. Main result}

Now, our main result can be presented. Considering the well-posedness of the kernel function in the transformation (44)-(47) together with the invertibility of the transformation, the following main theorem holds.

Theorem 1: Consider system (28)-(34) with boundary conditions (33)-(34), initial condition $\tilde{\alpha}_{1}^{0}, \tilde{\alpha}_{2}^{0}, \tilde{\beta}_{1}^{0}, \tilde{\beta}_{2}^{0} \in$ $\mathcal{L}_{2}([0,1], \mathbb{R})$ and $\check{Q}^{0} \in \mathbb{R}$, and with output injection gains $p_{1}, p_{2}, p_{3}$ and $p_{Q}$ defined by (55)-(56), respectively, where $P_{1}, P_{2}, P_{3}$ and $P_{Q}$ are given by (58)-(66), respectively. Then, its zero equilibrium is exponentially stable in the $\mathcal{L}_{2}$ sense.

Using the results of Section III, this theorem can be proved with standard arguments (see more details in [22]).

\section{EXPERIMENTAL VALIDATION}

In this section we show the results of the proposed backstepping-based observer with experimental data of a Rijke tube prototype. The observer (21)-(27) was numerically integrated using the HPDE solver for Matlab [33], where a twostep variant of the Lax-Friedrichs method is used. In order to ensure the numerical stability, the time and space steps were chosen such that the Courant-Friedrichs-Lewy condition is satisfied. Several simulation tests were performed in order to find the number of points of the temporal and spatial grid. For the parameters used in this work (see Table I), more than 100 points in the spatial grid and a sampling time of $0.0001 \mathrm{~s}$ did not show a significant change in the exponential convergence of the observer. The gains (55)-(56) were computed explicitly from expressions (58), (60), (62), and (63)-(64). Note that (21)-(27) represents the system in characteristic coordinates and therefore, the algebraic relations (5)-(6) and (12) must be applied to reconstruct the state variables in the original coordinates.

All the experiments were performed on a simple 1.3 meter long glass tube (see Figure 2) with an electrical heating element made of nichrome wire coil. This prototype was inspired by [34]. The power is delivered to the coil using a DC power supply with fixed power output of $360 \mathrm{~W}$. The location of the electrical heating element was chosen to be a quarter of the tube length. The boundary acoustic pressure in the top of the tube is measured with a clip-on microphone with builtin preamplifier. In addition, another clip-on microphone was installed at the middle of the tube in order to compare the behavior of the observer (21)-(27) with the actual values in that point of the domain. It is important to emphasize that this measurement is not used as an injection term in our design.

The measurements are sent to a control computer through a data acquisition (DAQ) device. In this context, the analog signals are conditioned and processed by the DAQ in order to be converted into digital signals and then, injected in the observer algorithm coded in Matlab. The interested reader is referred to [35] for more information on digital signal processing.

The microphone was calibrated using a sound level meter (SLM) and comparing the level of sound on the SLM with the signal values obtained from the microphone. The steadystate wire temperature was computed using an infrared thermometer. The other parameter values, such the steady-state 


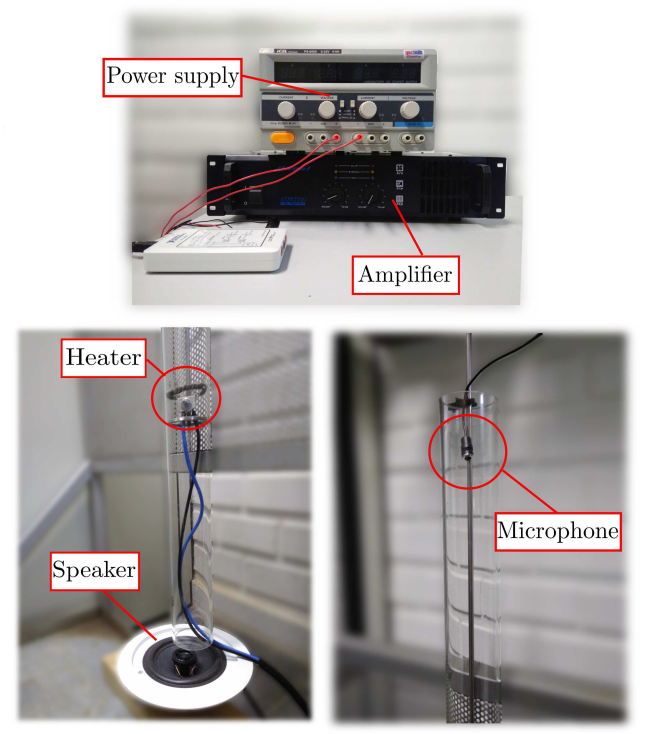

Fig. 2. Photograph of the Rijke tube prototype with highlights of its main components: the power supply, the linear amplifier, the heater element, the speaker (not used in this work) and the boundary pressure measurement.

TABLE I

VALUES OF THE PARAMETERS OF THE SYSTEM.

\begin{tabular}{lll}
\hline Symbol & Description & Value \\
\hline $\bar{\rho}$ & Steady-state density & $1.2 \mathrm{~kg} / \mathrm{m}^{3}$ \\
$\bar{P}$ & Steady-state pressure & $10^{5} \mathrm{~Pa}$ \\
$\bar{v}$ & Steady-state velocity & $0.5 \mathrm{~m} / \mathrm{s}$ \\
$\gamma$ & Adiabatic ratio & 1.4 \\
$\bar{\gamma}$ & - & 0.4 \\
$R$ & Ideal gas constant & $290 \mathrm{~J} /(\mathrm{kg} \mathrm{K})$ \\
$L$ & Tube length & $1.35 \mathrm{~m}$ \\
$l_{w}$ & Wire length & $1.4 \mathrm{~m}$ \\
$x_{0}$ & Heater position & $\frac{1}{4} L \mathrm{~m}$ \\
$d$ & Tube diameter & $0.0762 \mathrm{~m}$ \\
$d_{w}$ & Wire diameter & $0.003 \mathrm{~m}$ \\
$T_{w}$ & Wire temperature & $933 \mathrm{~K}$ \\
$Z_{L}$ & Acoustic impedance & $10.6095 \mathrm{~Pa} \mathrm{~s} / \mathrm{m}$ \\
$\tau_{h r}$ & Heat release time constant & $0.001 \mathrm{~s}$ \\
\hline
\end{tabular}

velocity, heat release time constant and acoustic impedance were estimated using some relations between other parameters and variables. As shown in [34], $\bar{v}$ can be approximated by $\bar{v}=\sqrt{g \frac{T_{w}-\bar{T}_{\text {gas }}}{\bar{T}_{\text {gas }}} d_{w}}$, where $g$ is the gravitational acceleration and $d_{w}$ is the diameter of the wire, and the heat release time constant is estimated as $\tau_{h r}=0.2 d_{w} / \bar{v}$ due to [36]. Finally, the acoustic impedance was computed via two microphones positioned near the boundary in the actual experiment (see [37] for more details about this procedure). The obtained parameter estimations are shown in Table I.

Measured and estimated acoustic pressure fluctuations at $x=L$ are shown in Figure 3, where the initial error in the acoustic pressure estimation is due to intentionally chosen, incorrect initialization of the states. As can be seen, the observer converges to the real acoustic pressure.

A comparison between the estimated and measured acoustic pressure fluctuations at $x=\frac{1}{2} L$ can be seen in Figure 4. As we can observe in that figure, the standing wave of both the model and the experiment are in good agreement, after the initial

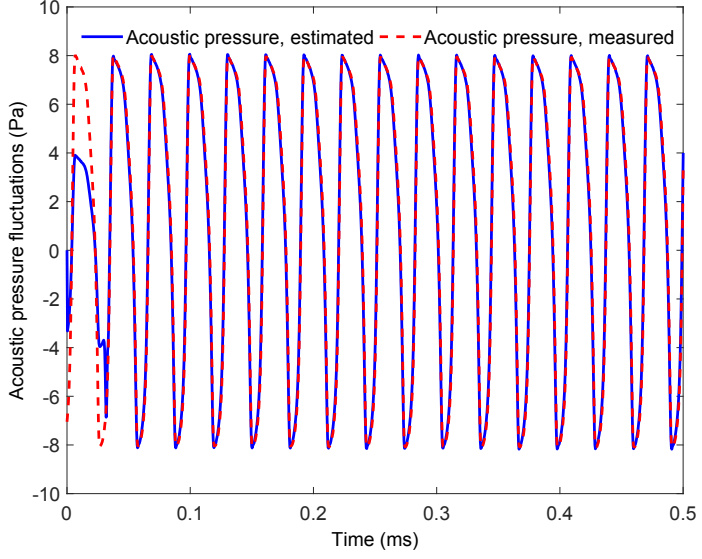

Fig. 3. Measured and estimated acoustic pressure at $x=L$.

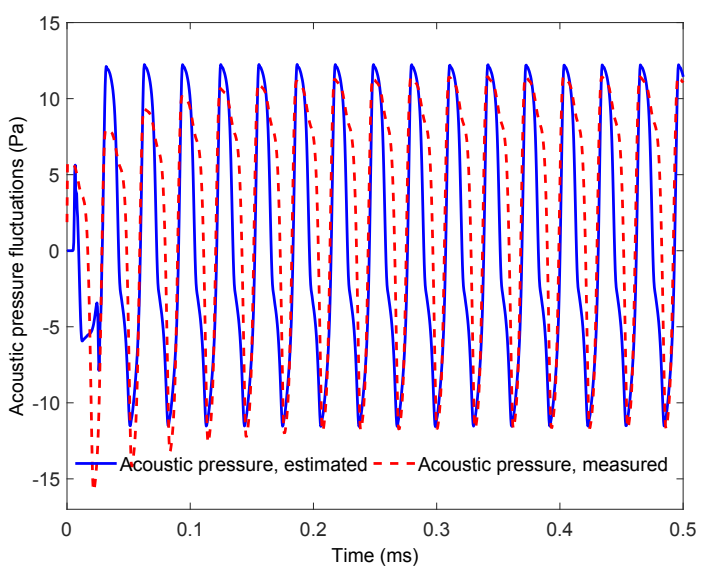

Fig. 4. Measured and estimated acoustic pressure at $x=\frac{1}{2} L$.

errors coming from incorrect initialization of the model. The small discrepancy between the harmonics of the model and the experiment is explained by the modeling simplifications and parameter estimation. In particular, the linearized model (1)-(4) was obtained assuming constant steady-state along the space (see [4]). In addition, the heat release dynamics is related only with the velocity fluctuations, whereas a full model would be related with the acoustic pressure. However, If these effects were taken into account in the linearized model, the computations of the observer would be much more involved. The strength of our observer design lies mainly in its simplicity and usability for control and optimization purposes, since the system (1)-(4) is capable of reproducing the main characteristics of the thermoacoustic phenomenon.

\section{CONCLUSION}

We have solved the boundary observer design problem for thermoacoustic instabilities in the Rijke tube. The design, which is based on the backstepping method, relies on only one boundary acoustic pressure measurement and the observer gains can be computed explicitly. The resulting kernels of the backstepping transformation are piecewise differentiable, with the number of pieces depending on the characteristic velocities of the system. To the best of our knowledge, this is the first result designing a Rijke Tube observer from an infinite 
dimensional perspective, which had to adapt the standard backstepping methodology due to the system being non-strictfeedback. Results with experimental data of a Rijke tube prototype were presented to show the effectiveness of the design.

Directions for further research include the extension of the method for a more general class of PDE-ODEs with non-strictfeedback connection and measurements at an arbitrary point of the domain. Combining the approach proposed in this paper with [22], [38] could be considered as the initial step for this case. Other direction of future work includes the design of an adaptive observer for this system, which can be usefull when parameters are unknown. Finally, we will combine the observer design proposed in this paper with the backstepping controller developed in [7] in order to produce real-life closedloop experiments.

\section{ACKNOWLEDGMENT}

The authors gratefully acknowledge Universidad de Sevilla for funding part of this work under its V-PPI US and financial support of the Spanish Ministerio de Ciencia, Innovación y Universidades under grant PGC2018-100680-B-C21.

\section{REFERENCES}

[1] A. M. Annaswamy and A. F. Ghoniem, "Active control in combustion systems," Control Systems Magazine, vol. 15, no. 6, pp. 49-63, 1995.

[2] A. Banaszuk, K. B. Ariyur, and M. Krstic, "An adaptive algorithm for control of combustion instability," Automatica, vol. 40, pp. 1965-1972, 2004.

[3] K. Kashinath, I. C. Waugh, and M. P. Juniper, "Nonlinear self-excited thermoacoustic oscillations of a ducted premixed flame: Bifurcations and routes to chaos," Journal of Fluid Mechanics, vol. 761, pp. 399-430, 2014.

[4] J. P. Epperlein, B. Bamieh, and J. Astrom, "Thermoacoustics and the Rijke tube: Experiments, identification and modeling," Control Systems Magazine, vol. 35, no. 2, pp. 57-77, 2015.

[5] G. A. de Andrade, R. Vazquez, and D. J. Pagano, "Boundary control of a rijke tube using irrational transfer functions with experimental validation," in Proceedings of the 20th IFAC World Congress, 2017, pp. 4528-4533.

[6] N. Bekiaris-Liberis and M. Krstic, "Stabilization of linear strict-feedback systems with delayed integrators," Automatica, vol. 46, pp. 1902-1910, 2010.

[7] G. A. de Andrade, R. Vazquez, and D. J. Pagano, "Backstepping stabilization of a linearized ODE-PDE rijke tube model," Automatica, vol. 96, pp. 98-109, 2018.

[8] A. Smyshlyaev and M. Krstic, "Boundary control of an anti-stable wave equation with anti-damping on the uncontrolled boundary," Systems \& Control Letters, vol. 58, pp. 617 - 623, 2009.

[9] H. Feng and B.-Z. Guo, "Output feedback stabilization of an unstable wave equation with general corrupted boundary observation," Automatica, vol. 50, pp. $3164-3172,2014$.

[10] W. Guo and B.-Z. Guo, "Parameter estimation and non-collocated adaptive stabilization for a wave equation subject to general boundary harmonic disturbance," IEEE Transactions on Automatic Control, vol. 58 , no. 7, pp. $1631-1643,2013$.

[11] D. Bresch-Pietri and M. Krstic, "Output-feedback adaptive control of a wave PDE with boundary anti-damping," Automatica, vol. 50, pp. 1407 $-1415,2014$

[12] M. Krstic, "Compensating actuator and sensor dynamics governed by diffusion PDEs," System \& Control Letters, vol. 58, pp. 372-377, 2009.

[13] S. Tang and C. Xie, "Stabilization for a coupled PDE-ODE control system," Journal of the Franklin Institute, vol. 348, pp. 2142 - 2155, 2011.

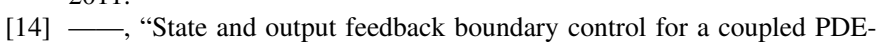
ODE system," Systems \& Control Letters, vol. 60, no. 8, pp. 540 - 545, 2011.
[15] N. Bekiaris-Liberis and M. Krstic, "Compensating the distributed effect of diffusion and counter-convection in multi-input and multi-output LTI systems," IEEE Transactions Automatic Control, vol. 56, pp. 637 - 643, 2011.

[16] M. Krstic and A. Smyshlyaev, "Backstepping boundary control for firstorder hyperbolic pdes and application to systems with actuator and sensor delays," Systems \& Control Letters, vol. 57, pp. 750 - 758, 2008.

[17] A. Hasan, O. M. Aamo, and M. Krstic, "Boundary observer design for hyperbolic PDE-ODE cascade systems," Automatica, vol. 68, pp. $75-$ 86, 2016.

[18] N. Bekiaris-Liberis and M. Krstic, "Compensating the distributed effect of a wave PDE in the actuation or sensing path of MIMO LTI systems," Systems \& Control Letters, vol. 59, pp. 713 - 719, 2010.

[19] M. Krstic and N. Bekiaris-Liberis, "Nonlinear stabilization in infinite dimension," Annual Reviews in Control, vol. 37, pp. 220-231, 2013.

[20] M. Krstic and A. Smyshlayeav, Boundary control of PDEs: a course on backstepping designs. SIAM, 2008.

[21] J.-M. Coron, L. Hu, and G. Olive, "Finite-time boundary stabilization of general linear hyperbolic balance laws via Fredholm backstepping transformation," Automatica, vol. 84, pp. 95 - 100, 2017.

[22] L. Hu, F. D. Meglio, R. Vazquez, and M. Krstic, "Control of homodirectional and general heterodirectional linear coupled hyperbolic PDEs," IEEE Transactions on Automatic Control, vol. 10, pp. 3301-3314, 2016.

[23] N. Bekiaris-Liberis and M. Krstic, "Lyapunov stability of linear predictor feedback for distributed input delays," IEEE Transactions on Automatic Control, vol. 56, pp. 655-660, 2011.

[24] C. Guo, C. Xie, and C. Zhou, "Stabilization of a spatially non-causal rection-diffusion equation by boundary control," International Journal of Robust and Nonlinear Control, vol. 24, pp. 1-17, 2012.

[25] P. Ascencio, A. Astolfi, and T. Parisini, "Backstepping PDE design: A convex optimization approach," IEEE Transactions on Automatic Control, vol. 63, pp. 1943 - 1958, 2018.

[26] G. A. de Andrade, R. Vazquez, and D. J. Pagano, "Backstepping-based linear boundary observer for estimation of thermoacoustic instabilities in a Rijke tube," 2018, , accepted to be presented in 57th IEEE Conference on Decision and Control.

[27] F. Di Meglio and U. J. F. Aarsnes, "A distributed parameter systems view of control problems in drilling," in Proceedings of the 2nd IFAC Workshop on Automatic Control in Offshore Oil and Gas Production, 2015, pp. 272-278.

[28] D. Maity, T. Takahashi, and M. Tucsnak, "Analysis of a system modelling the motion of a piston in a viscous gas," Journal of Mathematical Fluid Mechanics, vol. 19, pp. 551-579, 2017.

[29] G. A. de Andrade, J. D. Alvarez, D. J. Pagano, and M. Berenguel, "Nonlinear controllers for solar thermal plants: a comparative study," Control Engineering Practice, vol. 43, pp. 12-20, 2015.

[30] G. A. de Andrade, D. J. Pagano, J. L. Guzmán, M. Berenguel, I. Fernández, and F. G. Acién, "Distributed sliding mode control of ph in tubular photobioreactors," IEEE Transactions on Control Systems Technology, vol. 24, pp. 1160-1173, 2016.

[31] L. C. Evans, Partial differential equations. American Mathematical Society, 2010.

[32] R. Vazquez, E. Trélat, and J.-M. Coron, "Control for fast and stable laminar-to-high-Reynolds-numbers transfer in a 2D Navier-Stokes channel flow," Discrete Contin. Dyn. Syst. Ser. B, vol. 10, no. 4, pp. 925-956, 2008.

[33] L. F. Shampine, "Solving hyperbolic PDEs in MATLAB," Applied Numerical Analysis and Computational Mathematics Journal, vol. 2, pp. 346-358, 2015.

[34] J. P. Epperlein, "Topics in modeling and control of spatially distributed systems," Ph.D. dissertation, University of California, Santa Barba, USA, 2014, (in portuguese).

[35] G. F. Franklin, D. J. Powell, and M. L. Workman, Digital control of dynamic systems. Prentice-Hall, 1997.

[36] M. J. Lighthill, "The response of laminar skin friction and heat transfer to fluctuations in the stream velocity," Proceedings of the Royal Society A: Mathetical, Physical and Engineering Sciences, vol. 224, no. 1156, pp. $1-23,1954$.

[37] A. F. Seybert and D. F. Ross, "Experimental determination of acoustic properties using a two-microphone random-excitation technique," Journal of the Acoustical Society of America, vol. 61, pp. 1362 - 1370, 1977.

[38] J. Auriol, U. J. F. Aarsnes, P. Martin, and F. Di Meglio, "Delay-robust control design for two heterodirectional linear coupled hyperbolic pdes," IEEE Transactions on Automatic Control, vol. 63, p. 3551-3557, 2018. 